ARTIFICIAL SATELLITES, Vol. 44, No. 2 - 2009
DOI: 10.2478/v10018-009-0018-3

\title{
ON THE GEODETIC ROTATION OF THE MAJOR PLANETS, THE MOON AND THE SUN
}

\author{
G.I. Eroshkin, V.V. Pashkevich \\ Central (Pulkovo) Astronomical Observatory of RAS \\ Pulkovskoe shosse, 65/1, 196140, St.Petersburg, Russia \\ e-mails: eroshkin@gao.spb.ru, apeks@gao.spb.ru
}

\begin{abstract}
The problem of the geodetic (relativistic) rotation of the major planets, the Moon and the Sun was studied in the paper by Eroshkin and Pashkevich (2007) only for the components of the angular velocity vectors of the geodetic rotation, which are orthogonal to the plane of the fixed ecliptic J2000. This research represents an extension of the previous investigation to all the other components of the angular velocity vector of the geodetic rotation, with respect to the body-centric reference frame from Seidelmann et al. (2005).
\end{abstract}

Keywords: geodetic rotation, angular velocity vector, body-centric reference frame, the major planets, the Moon, the Sun, ephemeris, least-squares method, spectral analysis.

\section{INTRODUCTION}

The principal gravitational interaction between the major planets, the Moon and the Sun in every relativistic ephemeris is modeled by considering these bodies as non-rotating point masses.

Nevertheless, an ephemeris of the major bodies of the solar system, based on the relativistic equations of the orbital motion, contains data necessary for the calculation of the secular and periodic components of the angular velocity of the geodetic rotation of these bodies. In accordance with Landau and Lifshitz (1975), a geodetic rotation arises when a body, having non-zero moments of inertia, is orbiting in the Riemannian space of general relativity. The vector of the angular velocity of the geodetic rotation, which is the most essential relativistic component of the body rotational motion around the proper center of mass, has the following expression:

$$
\bar{\sigma}_{i}=\frac{1}{c^{2}} \sum_{j \neq i} \frac{G m_{j}}{\left|\bar{R}_{i}-\bar{R}_{j}\right|^{3}} \bar{R}_{i}-\bar{R}_{j} \times\left(\frac{3}{2} \dot{\bar{R}}_{i}-2 \dot{\bar{R}}_{j}\right) .
$$

Here $c$ is the velocity of light; $G$ is the gravitational constant; $m_{j}$ is the mass of a body $j ; \quad \bar{R}_{i}, \dot{\bar{R}}_{i}, \bar{R}_{j}, \dot{\bar{R}}_{j}$ are the vectors of the barycentric position and velocity of bodies $i$ and $j$. The symbol $\times$ means a vector product; the subscripts $i$ and $j$ correspond to the Moon, the major planets and the Sun. 
Figure 1 depicts the triangle, which is used to define the components of the angular velocity vector of the geodetic rotation for any body of the Solar system.

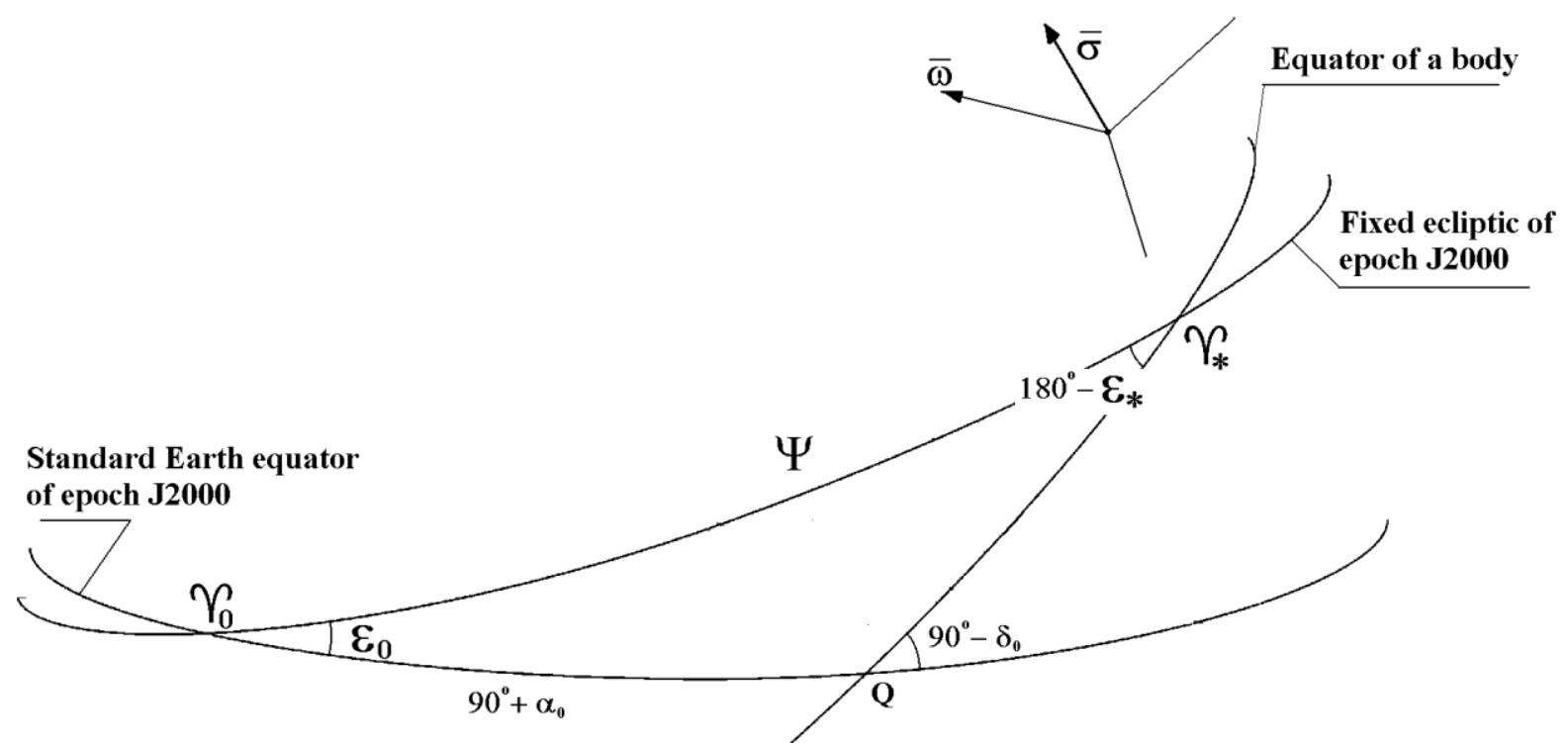

Fig. 1. Triangle used to define the components of the angular velocity vector of the geodetic rotation for any body

The reduction of the components of the angular velocity vector of the geodetic rotation of the solar system bodies, from the standard Earth equator J2000 (the reference frame of DE404/LE404 ephemeris) to the body-centric reference frames, given by Seidelmann et al. (2005), is presented by the following expressions:

from the standard Earth equator J2000 to the fixed ecliptic and the equinox J2000

$$
\begin{aligned}
& \sigma_{X_{0}}^{E c l}=\sigma_{X_{0}}^{E q u} \\
& \sigma_{Y_{0}}^{E c l}=\sigma_{Y_{0}}^{E q u} \cos \varepsilon_{0}+\sigma_{Z_{0}}^{E q u} \sin \varepsilon_{0} \\
& \sigma_{Z_{0}}^{E c l}=-\sigma_{Y_{0}}^{E q u} \sin \varepsilon_{0}+\sigma_{Z_{0}}^{E q u} \cos \varepsilon_{0},
\end{aligned}
$$

from the equinox J2000 to the ascending node of the equator of a body and the ecliptic J2000

$$
\begin{aligned}
& \cos \varepsilon_{*}=\cos \delta_{0} \sin \varepsilon_{0} \sin \alpha_{0}-\sin \delta_{0} \cos \varepsilon_{0} \\
& \sin \psi=\frac{\cos \delta_{0} \cos \alpha_{0}}{\sin \varepsilon_{*}} \\
& \sigma_{X_{*}}^{E c l}=\sigma_{X_{0}}^{E c l} \cos \psi+\sigma_{Y_{0}}^{E c l} \sin \psi \\
& \sigma_{Y_{*}}^{E c l}=-\sigma_{X_{0}}^{E c l} \sin \psi+\sigma_{Y_{0}}^{E c l} \cos \psi \\
& \sigma_{Z_{*}}^{E c l}=\sigma_{Z_{0}}^{E c l}
\end{aligned}
$$


from the ascending node of the equator of a body and the ecliptic J2000 to the equator of a body

$$
\begin{aligned}
\sigma_{X_{*}}^{E q u} & =\sigma_{X_{*}}^{E c l} \\
\sigma_{Y_{*}}^{E q u} & =\sigma_{Y_{*}}^{E c l} \cos \varepsilon_{*}-\sigma_{Z_{*}}^{E c l} \sin \varepsilon_{*} \\
\sigma_{Z_{*}}^{E q u} & =\sigma_{Y_{*}}^{E c l} \sin \varepsilon_{*}+\sigma_{Z_{*}}^{E c l} \cos \varepsilon_{*},
\end{aligned}
$$

where

$\sigma_{\psi}=\sigma_{Z_{*}}^{E c l}$ is the geodetic motion of the equator of a body on the fixed ecliptic J2000, $\sigma_{\theta}=\sigma_{X_{*}}^{E q u}$ is the geodetic variation of the obliquity of the equator of a body to the fixed ecliptic J2000,

$\sigma_{\varphi}=-\sigma_{Z_{*}}^{E q u}$ is the projection of the angular velocity vector of the geodetic rotation of a body to the axis of its angular velocity vector of the rotation $\bar{\omega}$,

$X, Y, Z$ are the components of the vector $\bar{R}$.

Here $\alpha_{0}$ is the right ascension of the north pole of the rotation of a body; $\delta_{0}$ is the declination of the north pole of rotation of a body. The values $\alpha_{0}$ and $\delta_{0}$ are taken from Seidelmann et al. (2005). The subscripts Ecl and Equ correspond to the ecliptic and the equator, respectively; $\sigma_{X}, \sigma_{Y}$ and $\sigma_{Z}$ are the projections of the angular velocity vector of the geodetic rotation on the axes of the reference frame.

\section{RAW DATA}

For each body the files with the data of the projections of the angular velocity vectors of the geodetic rotation on the axes of the body-centric reference frames are constructed over the time span from AD1000 to AD3000, with one day spacing, by using DE404/LE404 ephemeris. The behavior of the components of these angular velocity vectors of the geodetic rotation for the major planets, the Moon and the Sun are depicted in Figures $2-12$. In these Figures $\sigma_{\psi /}$ represents the geodetic motion of the equator of a body on the fixed ecliptic $\mathrm{J} 2000, \sigma_{\theta}$ describes the geodetic variation of the obliquity of the equator of a body to the fixed ecliptic J2000, $\sigma_{\varphi}$ represents the behavior of the projection of the angular velocity vector of the geodetic rotation of a body on the axis of the angular velocity vector of the rotation. 


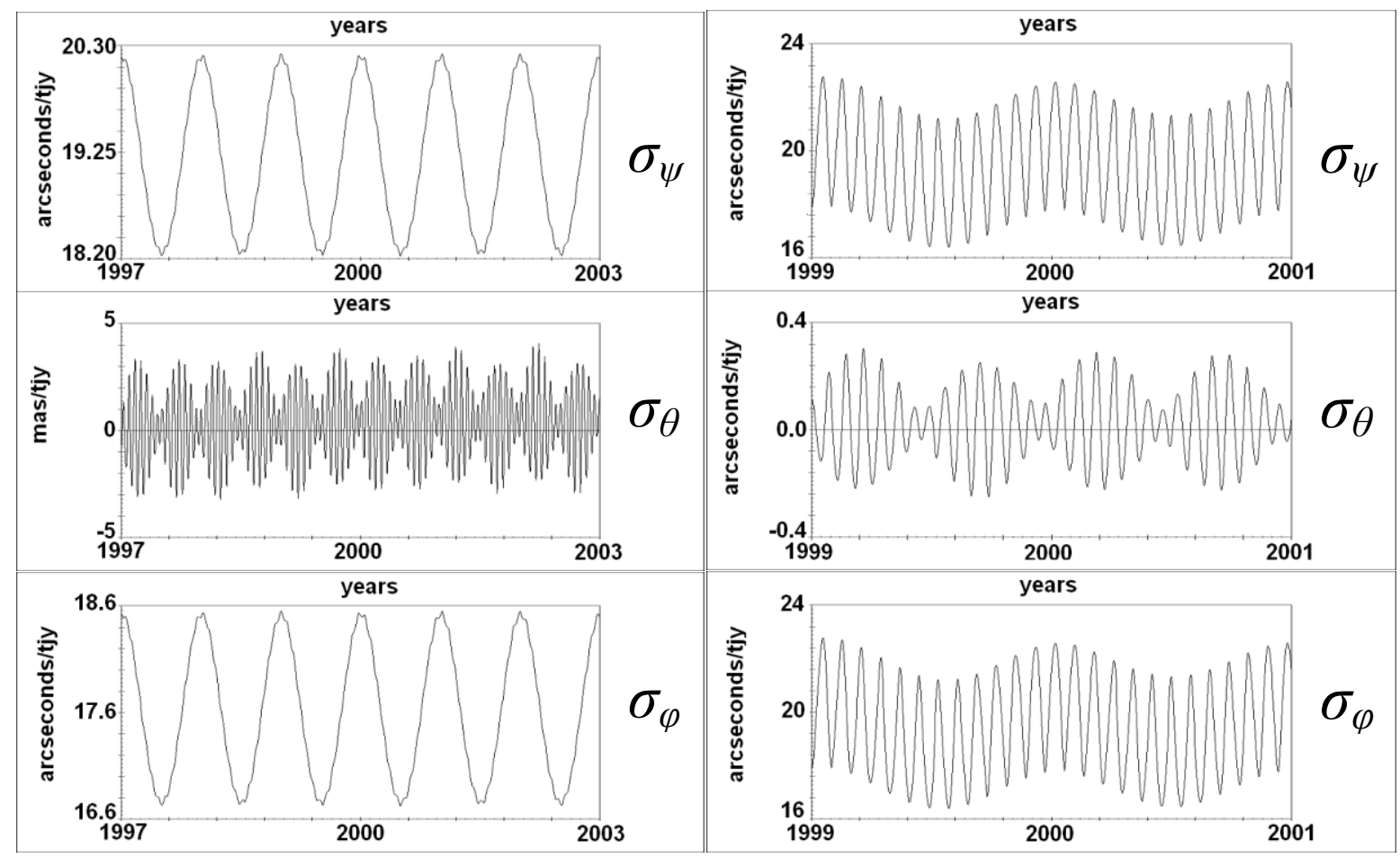

Fig. 2. For the Earth (fragment)

Fig. 3. For the Moon (fragment)

Since the mass of the Sun is dominant in the solar system then the main part of the angular velocity vector of the geodetic rotation $\bar{\sigma}$ for each major planet and the Moon is a result of the heliocentric orbital motion of these bodies. The geodetic rotation of the Moon is determined not only by the Sun but also by the Earth. Figure 3 demonstrates it visually.

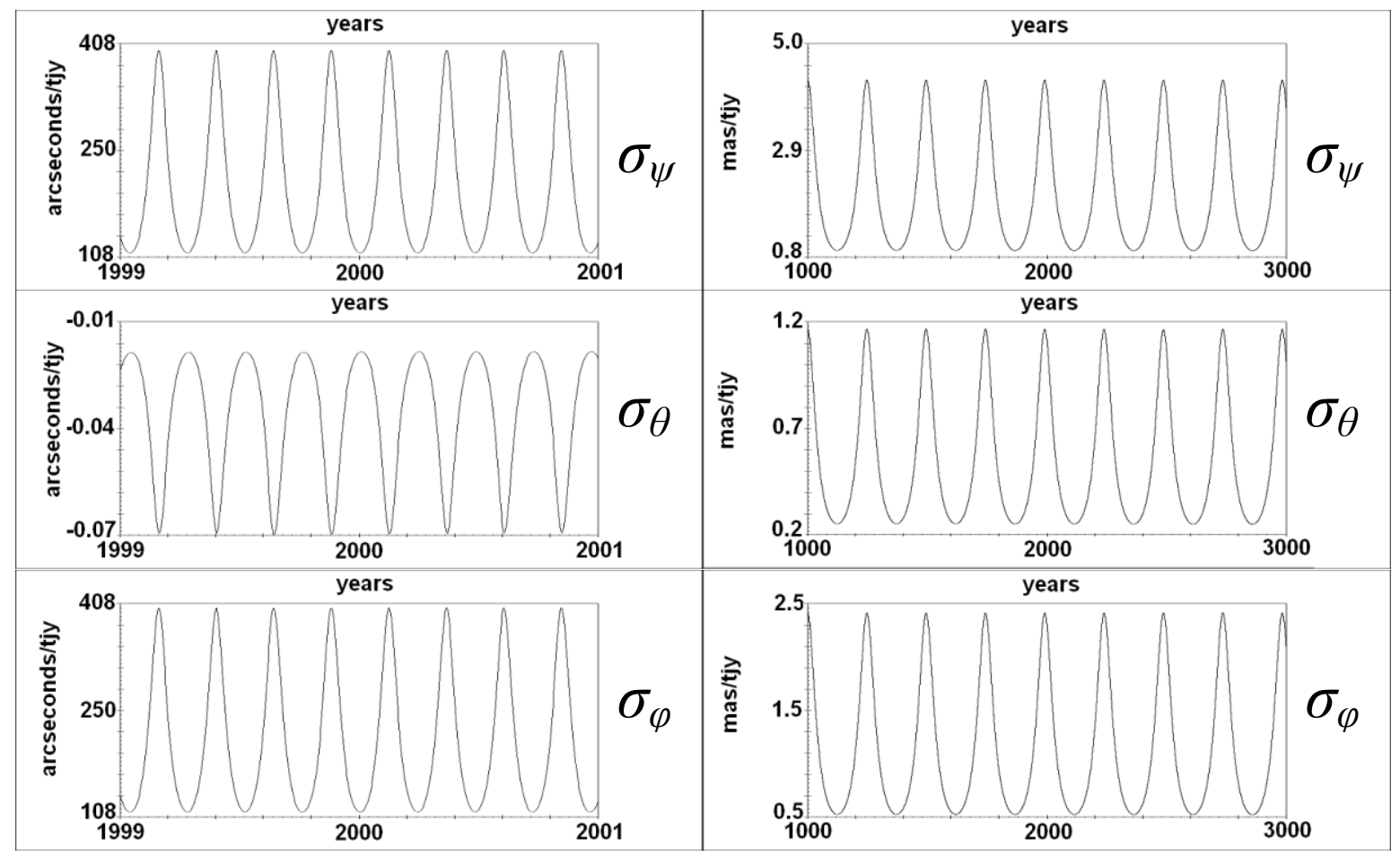

Fig. 4. For Mercury (fragment)

Fig. 5. For Pluto 
Some asymmetry of the components of the angular velocity vector of the geodetic rotation for Mercury (presented in Figure 4) and for Pluto (presented in Figure 5) is explained by the relatively large eccentricity of their orbits as compared to the other planet orbits (Table 1). The sharp peaks of the curve correspond to Mercury's and Pluto's transits via perihelia. The values of the eccentricities for Mercury orbit and for Pluto orbit are close to each other. This fact explains a similar character of the behavior of the components $\sigma_{\psi}$ and $\sigma_{\varphi}$ for these planets. Since Mercury is the nearest planet to the Sun then it is clear that its geodetic rotation has to be the most significant in the solar system.

Table 1 represents the main secular and periodic terms of the geodetic rotation in the longitude of the body equator, which are found by the integration of the corresponding values of the components $\sigma_{t /}$. Here and further $\lambda_{\mathrm{j}}(\mathrm{j}=1, \ldots 9)$ are the mean longitudes of the planets; $\lambda_{10}$ is the mean geocentric longitude of the Moon; $D=\lambda_{10}-\lambda_{3}+180^{\circ}$; T means the Dynamical Barycentric Time (TDB) measured in thousand Julian years (tjy) (of 365250 days) from J2000.

Table 1. The main secular and periodic terms of the geodetic rotation in the longitude of a body equator

\begin{tabular}{|c|c|c|c|}
\hline Object & Secular part & Periodic part & $\begin{array}{l}\text { Eccentricity } \\
\text { of the orbit }\end{array}$ \\
\hline Mercury & $213 " .300 \mathrm{~T}$ & $1078 " .175 \cdot 10^{-6} \sin \lambda_{1}-4845^{\prime \prime} .763 \cdot 10^{-6} \cos \lambda_{1}$ & 0.206 \\
\hline Venus & 43".048T & $-56 " .807 \cdot 10^{-6} \sin \lambda_{2}-64 " .066 \cdot 10^{-6} \cos \lambda_{2}$ & 0.007 \\
\hline The Earth & $19 " .199 \mathrm{~T}$ & $-34 " .283 \cdot 10^{-6} \sin \lambda_{3}-149^{\prime \prime} .221 \cdot 10^{-6} \cos \lambda_{3}$ & 0.017 \\
\hline $\begin{array}{l}\text { The } \\
\text { Moon }\end{array}$ & $19 " .494 \mathrm{~T}$ & $\begin{array}{r}-34^{\prime \prime} .278 \cdot 10^{-6} \sin \lambda_{3}-149^{\prime \prime} .200 \cdot 10^{-6} \cos \lambda_{3} \\
+30^{\prime \prime} .212 \cdot 10^{-6} \sin D\end{array}$ & \\
\hline Mars & $6 " .752 \mathrm{~T}$ & 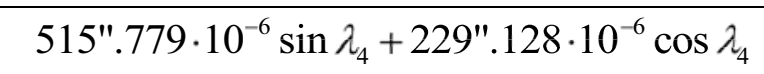 & 0.093 \\
\hline Jupiter & $0 " .312 \mathrm{~T}$ & $82 " .801 \cdot 10^{-6} \sin \lambda_{5}-21 " .288 \cdot 10^{-6} \cos \lambda_{5}$ & 0.048 \\
\hline Saturn & 0".069T & $-2^{\prime \prime} .691 \cdot 10^{-6} \sin \lambda_{6}-52 " .940 \cdot 10^{-6} \cos \lambda_{6}$ & 0.056 \\
\hline Uranus & $0 " .012 \mathrm{~T}$ & $-22 " .266 \cdot 10^{-6} \sin \lambda_{7}-3 " .466 \cdot 10^{-6} \cos \lambda_{7}$ & 0.046 \\
\hline Neptune & $0 " .004 \mathrm{~T}$ & $1 " .839 \cdot 10^{-6} \sin \lambda_{8}-1 " .780 \cdot 10^{-6} \cos \lambda_{8}$ & 0.009 \\
\hline Pluto & $0 " .002 \mathrm{~T}$ & $59 " .423 \cdot 10^{-6} \sin \lambda_{9}+0 " .273 \cdot 10^{-6} \cos \lambda_{9}$ & 0.249 \\
\hline the Sun & $0 " .001 \mathrm{~T}$ & $\begin{array}{r}0^{\prime \prime} .105 \cdot 10^{-6} \sin \lambda_{5}-0^{\prime \prime} .027 \cdot 10^{-6} \cos \lambda_{5} \\
-0 " .001 \cdot 10^{-6} \cos \lambda_{1}\end{array}$ & \\
\hline
\end{tabular}




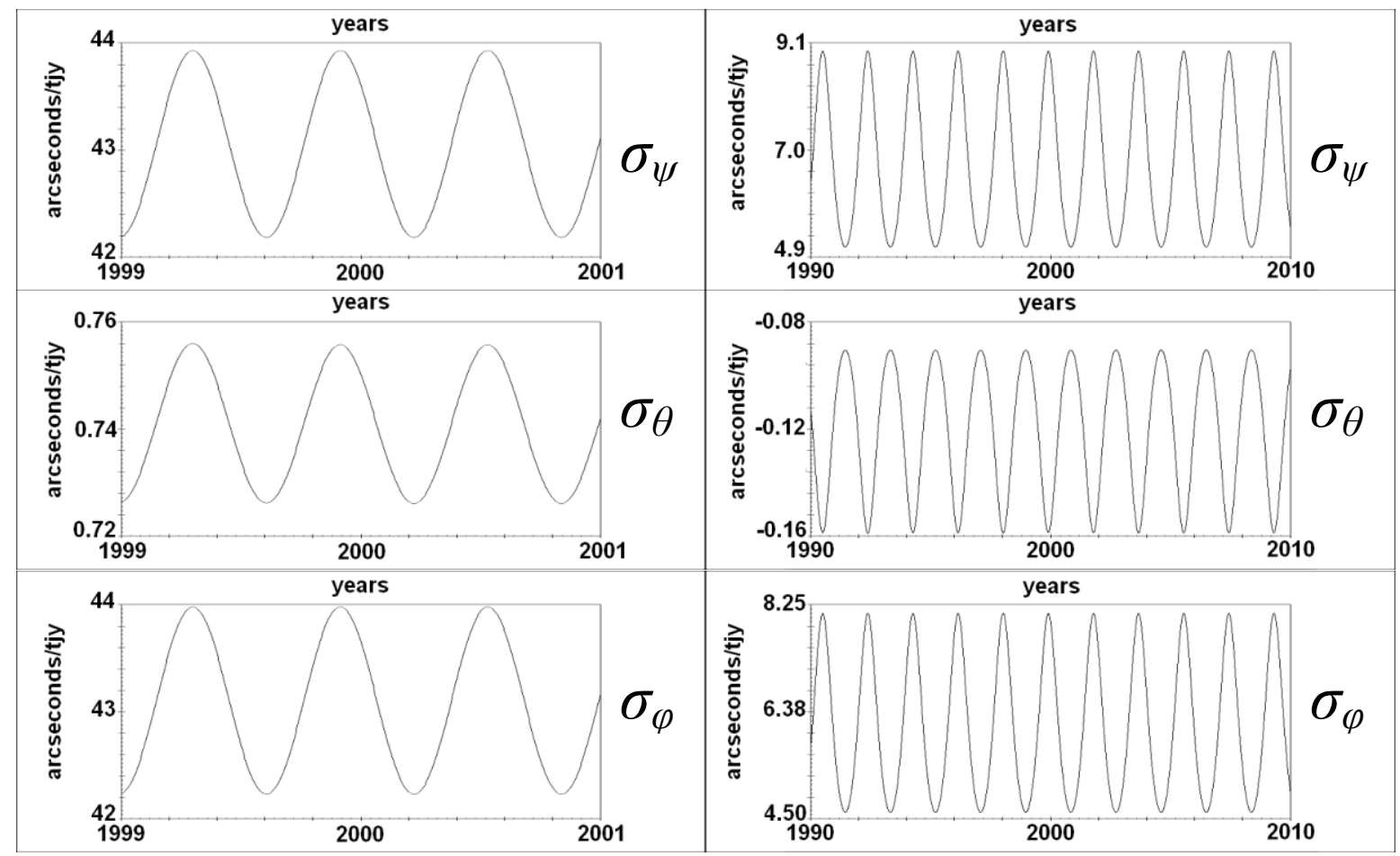

Fig. 6. For Venus (fragment)

Fig. 7. For Mars (fragment)

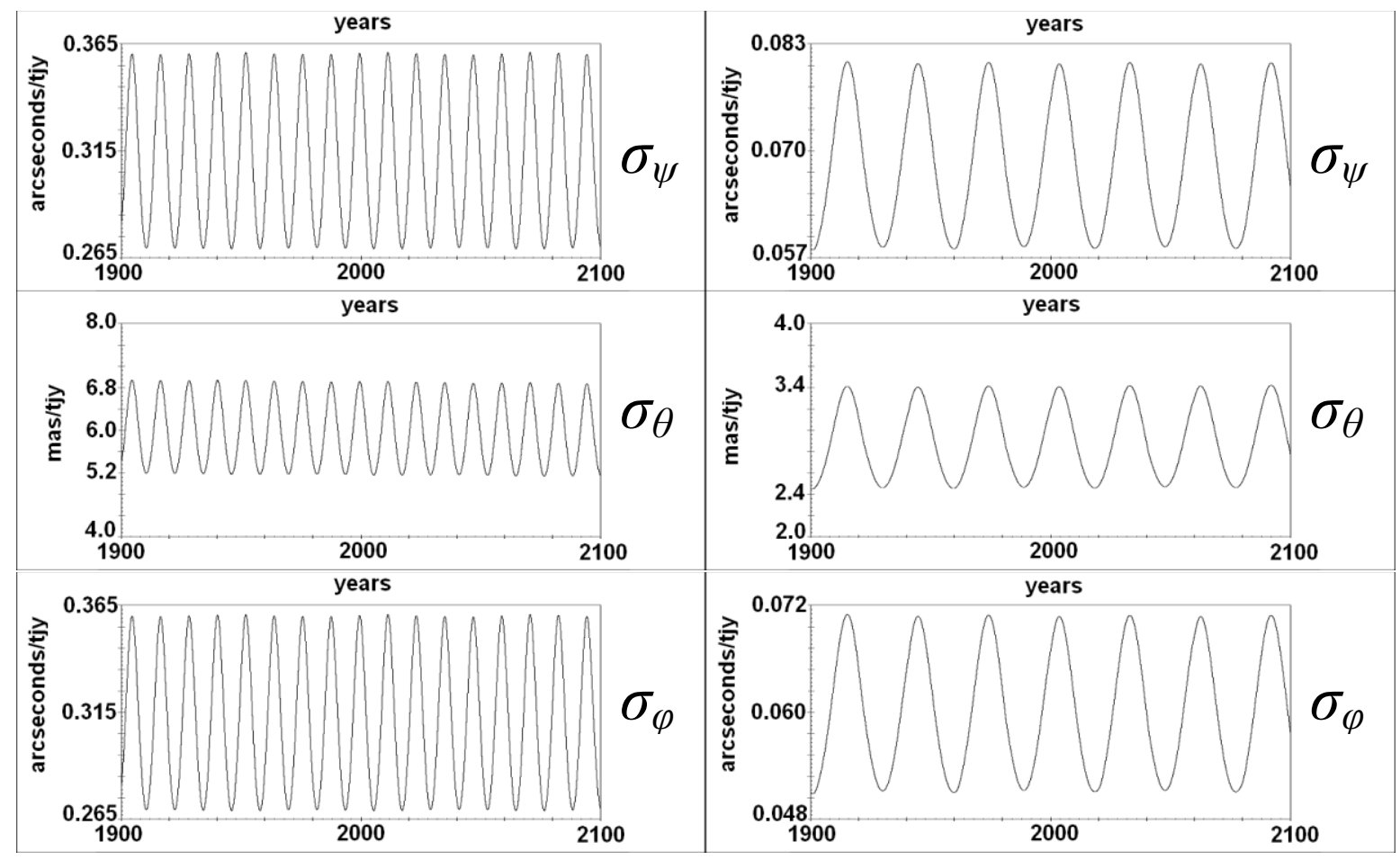

Fig. 8. For Jupiter (fragment)

Fig. 9. For Saturn (fragment) 


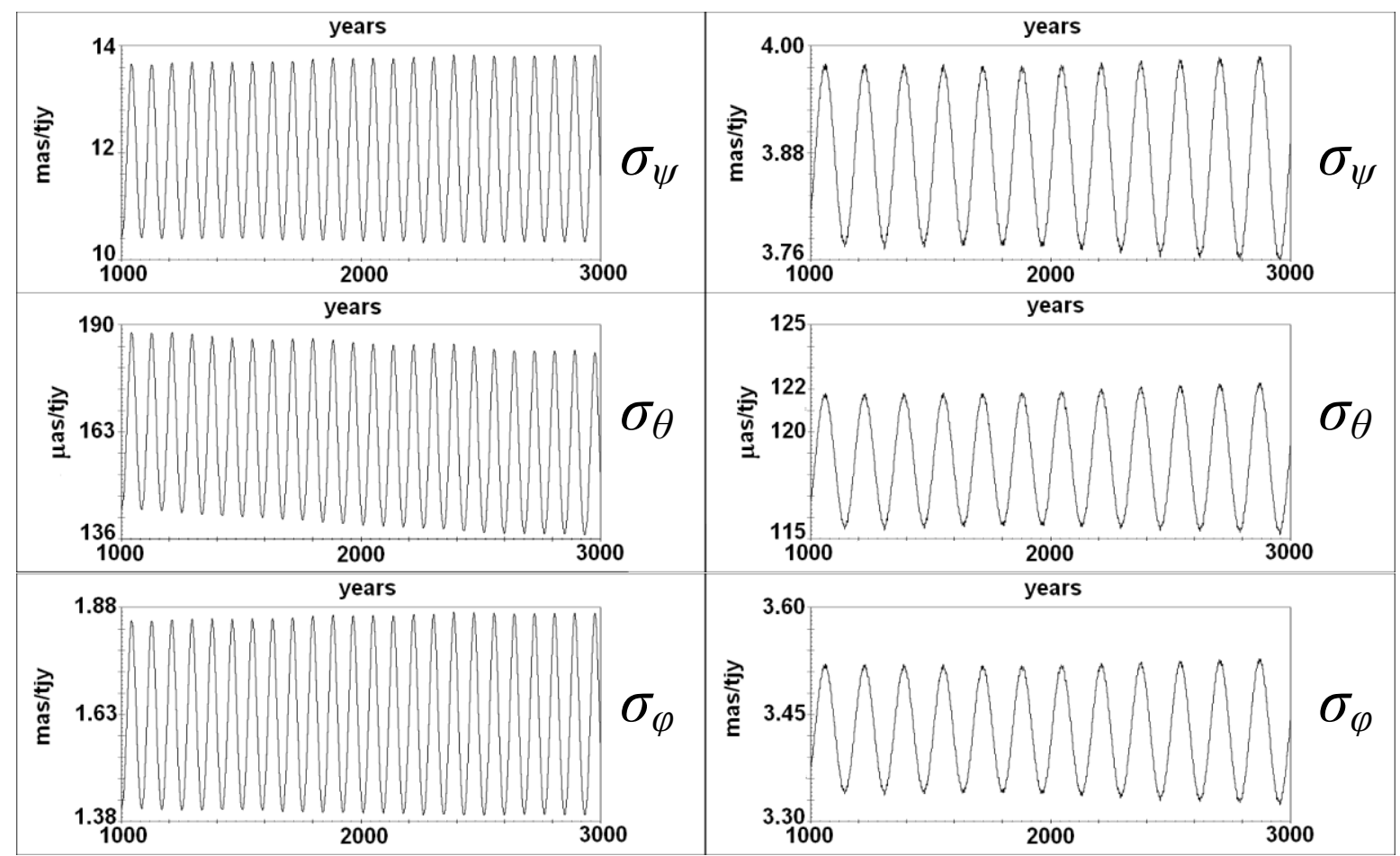

Fig. 10. For Uranus

Fig. 11. For Neptune

The position of the rotation axis of Uranus is close to the ecliptic plane. As a consequence, the difference between the values of the components $\sigma_{i /}$ and $\sigma_{\varphi}$ on Figure 10 is very significant as compared to the similar components for the other bodies.

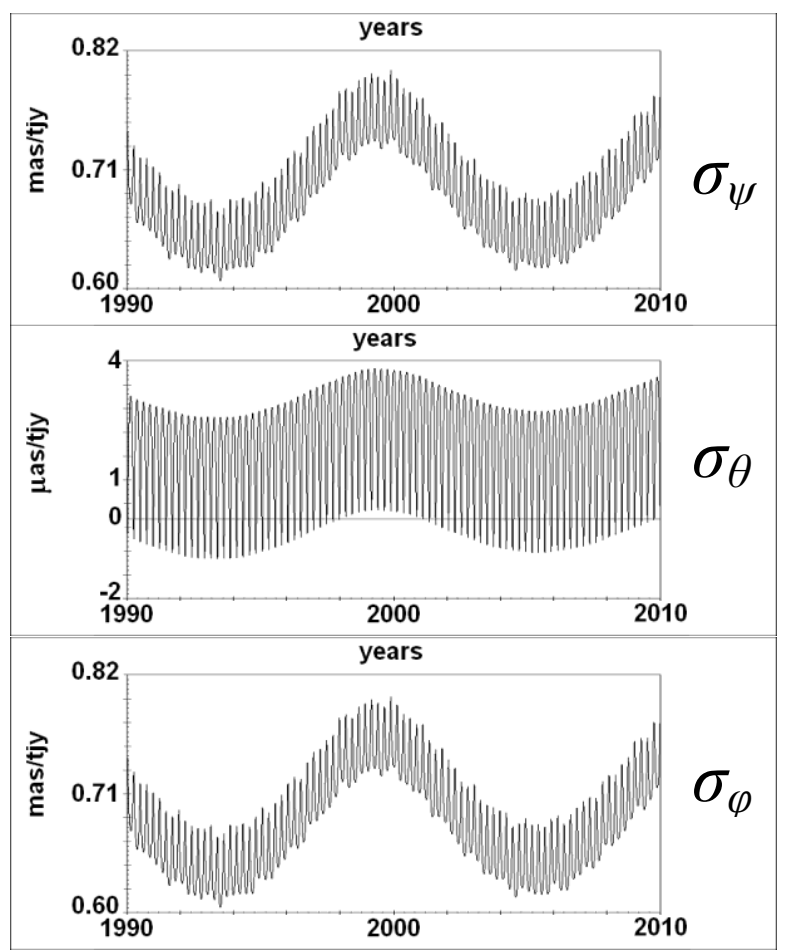

Fig. 12. For the Sun (fragment) 
The vector of the geodetic rotation of the Sun is determined by the orbital motion of the planets. Since the masses of the planets are essentially less than the mass of the Sun then the geodetic rotation of the Sun is very small. Its main part (presented in Figure 12) depends on the orbital motions of Jupiter, as the heaviest planet in the solar system, and Mercury, as the nearest planet to the Sun.

\section{RESULTS}

The most essential terms of the geodetic rotation are found by Eroshkin and Pashkevich (2007), by means of the least squares procedure and the spectral analysis methods. The mean longitudes of the planets, the Moon and Pluto, adjusted to the DE404/LE404 ephemeris, are taken from the previous investigation of Eroshkin and Pashkevich (2007).

The secular and periodic terms of the geodetic rotation of the solar system bodies for $\sigma_{\psi /}$, $\sigma_{\theta}$ and $\sigma_{p}$ are represented in the Table 2 and Table 3, respectively. It is easy to see that the values of the secular parts of the geodetic rotation of each planet depend on its distance from the Sun (Table 2). For any planet of the Solar system the periodic terms of the geodetic rotation depend not only on its distance from the Sun (Table 3), but also on the values of the eccentricities of their orbits (Table 1). So, since the value of the eccentricity for Mars is larger than that for the Earth then the amplitudes of the main periodic terms in the components of the geodetic rotation of Mars (Figure 7) are larger those that for the Earth (Figure 2).

Table 2. The secular terms of geodetic rotation for $\sigma_{\psi}, \sigma_{\theta}$ and $\sigma_{\varphi}$

\begin{tabular}{|c|c|c|c|c|}
\hline Mercury & $\begin{array}{l}213 " .3+0 " .050 \mathrm{~T}-0 " .029 \mathrm{~T}^{2}+\ldots \\
-0 " .036+0 " .006 \mathrm{~T}+6 " \cdot 10^{-4} \mathrm{~T}^{2+} \ldots \\
214 " .9+0 " .023 \mathrm{~T}-0 " .029 \mathrm{~T}^{2+} \ldots\end{array}$ & Jupiter & $\begin{array}{l}0 " .312-1^{\prime \prime} \cdot 10^{-4} \mathrm{~T}+0^{\prime \prime} .005 \mathrm{~T}^{2}+\ldots \\
0^{\prime \prime} .006-3^{\prime \prime} \cdot 10^{-4} \mathrm{~T}+5^{\prime \prime} \cdot 10^{-5} \mathrm{~T}^{2}+\ldots \\
0^{\prime \prime} .311-1^{"} \cdot 10^{-4} \mathrm{~T}+0^{\prime \prime} .005 \mathrm{~T}^{2}+\ldots\end{array}$ & $\begin{array}{l}\sigma_{\psi} \\
\sigma_{\theta} \\
\sigma_{\varphi}\end{array}$ \\
\hline Venus & $\begin{array}{l}43 " .048-8^{\prime \prime} \cdot 10^{-4} \mathrm{~T}+5^{\prime \prime} \cdot 10^{-4} \mathrm{~T}^{2}+\ldots \\
0 " .741 \quad-0 " .12 \mathrm{~T}-0^{\prime \prime} .002 \mathrm{~T}^{2}+\ldots \\
43^{\prime \prime} .091-1 " \cdot 10^{-4} \mathrm{~T}+4^{\prime \prime} \cdot 10^{-4} \mathrm{~T}^{2}+\ldots\end{array}$ & Saturn & 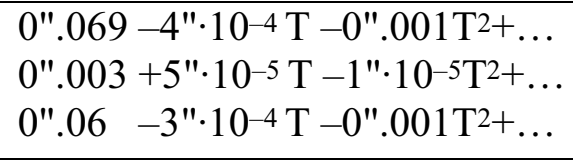 & $\begin{array}{l}\sigma_{\psi} \\
\sigma_{\theta} \\
\sigma_{\varphi}\end{array}$ \\
\hline $\begin{array}{l}\text { The } \\
\text { Earth }\end{array}$ & 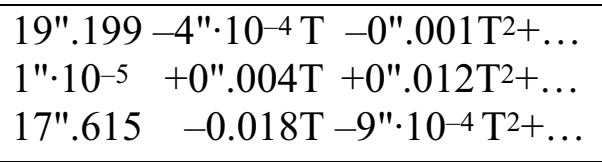 & Uranus & $\begin{array}{l}0^{\prime \prime} .012+2^{\prime \prime} \cdot 10^{-4} \mathrm{~T}+3 " \cdot 10^{-4} \mathrm{~T}^{2}+\ldots \\
2^{\prime \prime} \cdot 10^{-4}-1 " \cdot 10^{-6} \mathrm{~T}+4^{\prime \prime} \cdot 10^{-6} \mathrm{~T}^{2}+\ldots \\
0^{\prime \prime} .002+2^{\prime \prime} \cdot 10^{-5} \mathrm{~T}+5^{\prime \prime} \cdot 10^{-5} \mathrm{~T}^{2}+\ldots\end{array}$ & $\begin{array}{l}\sigma_{\psi} \\
\sigma_{\theta} \\
\sigma_{\varphi}\end{array}$ \\
\hline $\begin{array}{r}\text { The } \\
\text { Moon }\end{array}$ & $\begin{array}{l}19 " .494+1 " \cdot 10^{-5} \mathrm{~T}-0 " .002 \mathrm{~T}^{2}+\ldots \\
3 " \cdot 10^{-4}+0 " .004 \mathrm{~T}+0^{\prime \prime} .009 \mathrm{~T}^{2}+\ldots \\
19 " .494+9 " \cdot 10^{-6} \mathrm{~T}-0 " .002 \mathrm{~T}^{2}+\ldots\end{array}$ & Neptune & $\begin{array}{l}0^{\prime \prime} .004-3^{\prime \prime} \cdot 10^{-5} \mathrm{~T}-4^{\prime \prime} \cdot 10^{-5} \mathrm{~T}^{2}+\ldots \\
1 " \cdot 10^{-4}-1^{\prime \prime} \cdot 10^{-6} \mathrm{~T}-1 " \cdot 10^{-6} \mathrm{~T}^{2}+\ldots \\
0^{\prime \prime} .003-2^{\prime \prime} \cdot 10^{-5} \mathrm{~T}-4^{\prime \prime} \cdot 10^{-5} \mathrm{~T}^{2}+\ldots\end{array}$ & $\begin{array}{l}\sigma_{\psi} \\
\sigma_{\theta} \\
\sigma_{\varphi}\end{array}$ \\
\hline Mars & $\begin{array}{l}6 " .752+2 " \cdot 10^{-5} \mathrm{~T}-0 " .026 \mathrm{~T}^{2}+\ldots \\
-0 " .12+0 " .002 \mathrm{~T}+6 " \cdot 10^{-4} \mathrm{~T}^{2}+\ldots \\
6 " .113-0 " .007 \mathrm{~T}-0 " .023 \mathrm{~T}^{2}+\ldots\end{array}$ & Pluto & $\begin{array}{l}0^{\prime \prime} .002+5^{"} \cdot 10^{-5} \mathrm{~T}+\ldots \\
5^{\prime \prime} \cdot 10^{-4}+1 " \cdot 10^{-5} \mathrm{~T}+\ldots \\
0^{\prime \prime} .001+3^{\prime \prime} \cdot 10^{-5} \mathrm{~T}+\ldots\end{array}$ & $\begin{array}{l}\sigma_{\psi} \\
\sigma_{\theta} \\
\sigma_{\varphi}\end{array}$ \\
\hline & The Sun & \multicolumn{2}{|c|}{$\begin{array}{l}7^{\prime \prime} \cdot 10^{-4}-3^{\prime \prime} \cdot 10^{-7} \mathrm{~T}+6^{\prime \prime} \cdot 10^{-6} \mathrm{~T}^{2}+\ldots \\
2^{\prime \prime} \cdot 10^{-6}-2^{\prime \prime} \cdot 10^{-7} \mathrm{~T}+9^{\prime \prime} \cdot 10^{-10} \mathrm{~T}^{2}+\ldots \\
7^{\prime \prime} \cdot 10^{-4}-3^{\prime \prime} \cdot 10^{-7} \mathrm{~T}+6^{\prime \prime} \cdot 10^{-6} \mathrm{~T}^{2}+\ldots\end{array}$} & $\begin{array}{l}\sigma_{\psi} \\
\sigma_{\theta} \\
\sigma_{\varphi}\end{array}$ \\
\hline
\end{tabular}


Table 3. The periodic terms of geodetic rotation for $\sigma_{\psi}, \sigma_{\theta}$ and $\sigma_{\varphi}$

\begin{tabular}{|c|c|c|}
\hline Mercury & $\begin{array}{l}\left((1078 " .175-133 " .366 \mathrm{~T}+\ldots) \cos \lambda_{1}+\left(4845 " .763+35^{\prime \prime} .597 \mathrm{~T}+\ldots\right) \sin \lambda_{1}+\ldots\right) \cdot 10^{-6} \\
\left((-0 " .182+0 " .053 \mathrm{~T}+\ldots) \cos \lambda_{1}+\left(-0^{\prime} .819+0 " .128 \mathrm{~T}+\ldots\right) \sin \lambda_{1}+\ldots\right) \cdot 10^{-6} \\
\left((1086 " .284-134 " .507 \mathrm{~T}+\ldots) \cos \lambda_{1}+\left(4882 " .206+35^{\prime \prime} .241 \mathrm{~T}+\ldots\right) \sin \lambda_{1}+\ldots\right) \cdot 10^{-6}\end{array}$ & $\begin{array}{l}\sigma_{\psi} \\
\sigma_{\theta} \\
\sigma_{\varphi}\end{array}$ \\
\hline Venus & $\begin{array}{l}\left((-56 " .807+3 " .951 \mathrm{~T}+\ldots) \cos \lambda_{2}+\left(64 " .066-4^{\prime \prime} .565 \mathrm{~T}+\ldots\right) \sin \lambda_{2}+\ldots\right) \cdot 10^{-6} \\
\left((-0 " .978+0 " .227 \mathrm{~T}+\ldots) \cos \lambda_{2}+(1 " .103-0 " .258 \mathrm{~T}+\ldots) \sin \lambda_{2}+\ldots\right) \cdot 10^{-6} \\
\left((-56 " .864+3 " .954 \mathrm{~T}+\ldots) \cos \lambda_{2}+\left(64 " .13-4^{\prime \prime} .569 \mathrm{~T}+\ldots\right) \sin \lambda_{2}+\ldots\right) \cdot 10^{-6}\end{array}$ & $\begin{array}{l}\sigma_{\psi} \\
\sigma_{\theta} \\
\sigma_{\varphi}\end{array}$ \\
\hline The Earth & $\begin{array}{l}\left(\left(-34 " .283-7^{\prime \prime} .54 \mathrm{~T}+\ldots\right) \cos \lambda_{3}+\left(149^{\prime \prime} .221-5^{\prime \prime} .682 \mathrm{~T}+\ldots\right) \sin \lambda_{3}+\ldots\right) \cdot 10^{-6} \\
\left(\left(3^{\prime \prime} \cdot 10^{-5}-0^{\prime \prime} .007 \mathrm{~T}+\ldots\right) \cos \lambda_{3}+\left(2^{\prime \prime} \cdot 10^{-5}+0^{\prime \prime} .03 \mathrm{~T}+\ldots\right) \sin \lambda_{3}+\ldots\right) \cdot 10^{-6} \\
\left((-31 " .454-6 " .886 \mathrm{~T}+\ldots) \cos \lambda_{3}+\left(136^{\prime \prime} .907-5^{\prime \prime} .348 \mathrm{~T}+\ldots\right) \sin \lambda_{3}+\ldots\right) \cdot 10^{-6}\end{array}$ & $\begin{array}{l}\sigma_{\psi} \\
\sigma_{\theta} \\
\sigma_{\varphi}\end{array}$ \\
\hline The Moon & $\begin{array}{l}\left((-34 " .278-7 " .559 \mathrm{~T}+\ldots) \cos \lambda_{3}+(149 " .2-5 " .684 \mathrm{~T}+\ldots) \sin \lambda_{3}+\right. \\
\left.(30 " .212-0 " .001 \mathrm{~T}+\ldots) \cos D+\left(-7^{\prime \prime} \cdot 10^{-4}-0^{\prime \prime} .001 \mathrm{~T}+\ldots\right) \sin D+\ldots\right) \cdot 10^{-6} \\
\left(\left(-9 " \cdot 10^{-4}-0^{\prime \prime} .008 \mathrm{~T}+\ldots\right) \cos \lambda_{3}+\left(3^{\prime \prime} \cdot 10^{-4}+0^{\prime \prime} .025 \mathrm{~T}+\ldots\right) \sin \lambda_{3}+\right. \\
\left.\left(-0^{\prime \prime} .004-0 " .01 \mathrm{~T}+\ldots\right) \cos D+\left(-0^{\prime \prime} .005-0^{\prime \prime} .007 \mathrm{~T}+\ldots\right) \sin D+\ldots\right) \cdot 10^{-6} \\
\left((-34 " .278-7 " .559 \mathrm{~T}+\ldots) \cos \lambda_{3}+\left(149^{\prime \prime} .2-5^{\prime \prime} .684 \mathrm{~T}+\ldots\right) \sin \lambda_{3}+\right. \\
\left.\left(30^{\prime \prime} .212-0 " .001 \mathrm{~T}+\ldots\right) \cos D+\left(-7^{\prime \prime} \cdot 10^{-4}-0^{\prime \prime} .001 \mathrm{~T}+\ldots\right) \sin D+\ldots\right) \cdot 10^{-6}\end{array}$ & $\sigma_{\psi}$ \\
\hline Mars & $\begin{array}{l}\left(\left(515^{\prime \prime} .779+22 " .816 \mathrm{~T}+\ldots\right) \cos \lambda_{4}+(-229 " .128+37 " .702 \mathrm{~T}+\ldots) \sin \lambda_{4}+\ldots\right) \cdot 10^{-6} \\
\left(\left(-9 " .157-0^{\prime \prime} .241 \mathrm{~T}+\ldots\right) \cos \lambda_{4}+\left(4^{\prime \prime} .068-0^{\prime \prime} .742 \mathrm{~T}+\ldots\right) \sin \lambda_{4}+\ldots\right) \cdot 10^{-6} \\
\left((466 " .966+20 " .149 \mathrm{~T}+\ldots) \cos \lambda_{4}+(-207 " .443+34 " .359 \mathrm{~T}+\ldots) \sin \lambda_{4}+\ldots\right) \cdot 10^{-6}\end{array}$ & $\begin{array}{l}\sigma_{\psi} \\
\sigma_{\theta} \\
\sigma_{\varphi}\end{array}$ \\
\hline Jupiter & $\begin{array}{l}\left((82 " .801+1 " .961 \mathrm{~T}+\ldots) \cos \lambda_{5}+(21 " .288+3 " .999 \mathrm{~T}+\ldots) \sin \lambda_{5}+\ldots\right) \cdot 10^{-6} \\
\left((1 " .586-0 " .04 \mathrm{~T}+\ldots) \cos \lambda_{5}+\left(0^{\prime \prime} .408+0^{\prime \prime} .057 \mathrm{~T}+\ldots\right) \sin \lambda_{5}+\ldots\right) \cdot 10^{-6} \\
\left((82 " .699+1 " .958 \mathrm{~T}+\ldots) \cos \lambda_{5}+(21 " .261+3 " .994 \mathrm{~T}+\ldots) \sin \lambda_{5}+\ldots\right) \cdot 10^{-6}\end{array}$ & $\begin{array}{l}\sigma_{\psi} \\
\sigma_{\theta} \\
\sigma_{\varphi}\end{array}$ \\
\hline Saturn & $\begin{array}{l}\left((-2 " .691-5 " .173 \mathrm{~T}+\ldots) \cos \lambda_{6}+\left(52^{\prime \prime} .94-3^{\prime \prime} .494 \mathrm{~T}+\ldots\right) \sin \lambda_{6}+\ldots\right) \cdot 10^{-6} \\
\left(\left(-0 " .113-0^{\prime \prime} .222 \mathrm{~T}+\ldots\right) \cos \lambda_{6}+\left(2^{\prime \prime} .24-0^{\prime \prime} .099 \mathrm{~T}+\ldots\right) \sin \lambda_{6}+\ldots\right) \cdot 10^{-6} \\
\left(\left(-2^{2} .362-4 " .544 \mathrm{~T}+\ldots\right) \cos \lambda_{6}+(46 " .473-3 " .022 \mathrm{~T}+\ldots) \sin \lambda_{6}+\ldots\right) \cdot 10^{-6}\end{array}$ & $\begin{array}{l}\sigma_{\psi} \\
\sigma_{\theta} \\
\sigma_{\varphi}\end{array}$ \\
\hline Uranus & $\begin{array}{l}\left(\left(-22 " .266-1^{\prime \prime} .853 \mathrm{~T}+\ldots\right) \cos \lambda_{7}+\left(3^{\prime \prime} .466-0^{\prime \prime} .881 \mathrm{~T}+\ldots\right) \sin \lambda_{7}+\ldots\right) \cdot 10^{-6} \\
\left(\left(-0^{\prime \prime} .3-0^{\prime \prime} .018 \mathrm{~T}+\ldots\right) \cos \lambda_{7}+\left(0^{\prime \prime} .047-0^{\prime \prime} .013 \mathrm{~T}+\ldots\right) \sin \lambda_{7}+\ldots\right) \cdot 10^{-6} \\
\left(\left(-3 " .011-0^{\prime \prime} .247 \mathrm{~T}+\ldots\right) \cos \lambda_{7}+\left(0^{\prime \prime} .469-0^{\prime \prime} .12 \mathrm{~T}+\ldots\right) \sin \lambda_{7}+\ldots\right) \cdot 10^{-6}\end{array}$ & $\begin{array}{l}\sigma_{\psi} \\
\sigma_{\theta} \\
\sigma_{\varphi}\end{array}$ \\
\hline Neptune & $\begin{array}{l}\left((1 " .839+0 " .219 \mathrm{~T}+\ldots) \cos \lambda_{8}+\left(1 " .78+0^{\prime \prime} .302 \mathrm{~T}+\ldots\right) \sin \lambda_{8}+\ldots\right) \cdot 10^{-6} \\
\left(\left(0^{\prime \prime} .057+0 " .007 \mathrm{~T}+\ldots\right) \cos \lambda_{8}+\left(0^{\prime \prime} .055+0^{\prime \prime} .009 \mathrm{~T}+\ldots\right) \sin \lambda_{8}+\ldots\right) \cdot 10^{-6} \\
\left((1 " .627+0 " .194 \mathrm{~T}+\ldots) \cos \lambda_{8}+\left(1 " .574+0^{\prime \prime} .267 \mathrm{~T}+\ldots\right) \sin \lambda_{8}+\ldots\right) \cdot 10^{-6}\end{array}$ & \\
\hline Pluto & $\begin{array}{l}\left((59 " .423-2 " .028 \mathrm{~T}+\ldots) \cos \lambda_{9}+\left(-0^{\prime \prime} .273-13 " .109 \mathrm{~T}+\ldots\right) \sin \lambda_{9}+\ldots\right) \cdot 10^{-6} \\
\left(\left(16 " .174-0^{\prime \prime} .564 \mathrm{~T}+\ldots\right) \cos \lambda_{9}+\left(-0^{\prime \prime} .076-3^{\prime \prime} .564 \mathrm{~T}+\ldots\right) \sin \lambda_{9}+\ldots\right) \cdot 10^{-6} \\
\left((33 " .444-1 " .119 \mathrm{~T}+\ldots) \cos \lambda_{9}+\left(-0^{\prime \prime} .153-7^{\prime \prime} .378 \mathrm{~T}+\ldots\right) \sin \lambda_{9}+\ldots\right) \cdot 10^{-6}\end{array}$ & \\
\hline The Sun & 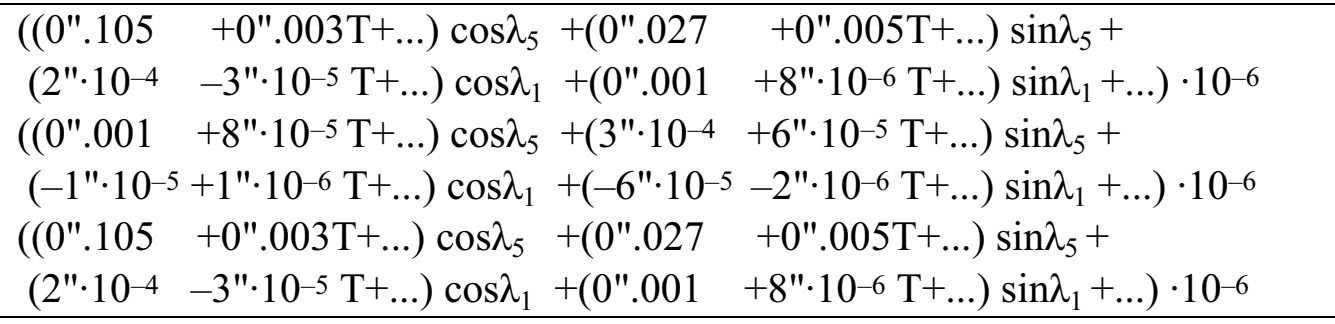 & $\begin{array}{c}\sigma_{\psi} \\
\sigma_{\theta}\end{array}$ \\
\hline
\end{tabular}




\section{CONCLUSIONS}

The secular terms of geodetic rotation of each planet of the Solar system depend on their distance from the Sun, the mass of which is dominant in the solar system. For any planet the periodic part of the geodetic rotation depends on its distance from the Sun and from the values of the eccentricities of their orbits. The main periodic part of the geodetic rotation of the Moon is determined not only by the Sun but also by the Earth, which is the nearest planet to the Moon. The geodetic rotation of the Sun depends not only on the orbital motion of Jupiter, as the heaviest planet in the solar system, but also on the orbital motion of Mercury which has the most rapid orbital motion.

\section{PERSPECTIVES}

For the terrestrial planets and the Moon the geodetic rotation is significant and has to be taken into account for the construction of the high-precision theories of the rotational motion of these bodies at a long time interval. The geodetic rotation has to be taken into account if the influence of the dynamical figure of a body on its orbital-rotational motion is studied in the post-Newtonian approximation. The lunar laser ranging data processing has to use the relativistic theory of the rotation of the Moon, as well as that of the Earth.

\section{ACKNOWLEDGEMENTS}

This investigation was carried out at the Central (Pulkovo) Astronomical Observatory of the Russian Academy of Science and the Space Research Centre of the Polish Academy of Science, under a financial support of the Cooperation between the Polish Academy of Science and the Russian Academy of Science, Theme No 38.

\section{REFERENCES}

Landau L.D. and Lifshitz E.M. (1975): The Classical Theory of Fields, Oxford: Pergamon Press.

Seidelmann P.K., Archinal B.A., A'Hearn M.F., Cruikshank D.P., Hilton J.L., Keller H.U., Oberst J., Simon J.L., Stooke P., Tholen D.J., and Thomas P.C. (2005): Report of the IAU/IAG Working Group on Carto-graphic Coordinates and Rotational Elements: 2003, Celestial Mechanics and Dynamical Astronomy, 91, pp. 203-215.

Eroshkin G.I., Pashkevich V.V. (2007): Geodetic rotation of the Solar system bodies, Artificial Satellites, Vol. 42, No. 1, pp. 59-70.

Received: 2009-09-23,

Reviewed: 2009-12-07,

Accepted: 2009-12-08. 